\section{Highlights of the San Antonio Breast Cancer Symposium 2016: Part 2}

\author{
John R Benson*,1 \& Ismail Jatoi \\ 39th annual San Antonio Breast Cancer Symposium \\ San Antonio, Texas, USA, 9-13 December 2016
}

The 39th annual San Antonio Breast Cancer Symposium (SABCS) was convened in San Antonio, Texas, on 9-13 December 2016. More than 7000 clinicians and scientists from around the world participated in the symposium which featured a range of presentations and keynote talks pertaining to breast cancer screening, prevention, locoregional and systemic therapies. This two-part report highlights a selection of important studies presented at this premier breast cancer event with Part 1 focusing onmetastatic breast cancer, extended endocrine therapy and the prognostic significance of BRCA $1 / 2$ gene mutations. The second part of this report will discuss a range of topics including anti-HER2 directed treatments, the impact of radiotherapy on implant and autologous flap based reconstruction, biological risk predictors for ductal carcinoma-in situ (DCIS), longer term effects of dietary fat modification and the influence of aromatase inhibitors on endothelial cell function.

First draft submitted: 27 February 2017; Accepted for publication: 20 April 2017; Published online: 7 July

First results of the PAMELA trial (NCT 01973660) were discussed by Aleix Prat (University of Barcelona, Spain). This nonrandomized open-label multicenter trial examined the PAM50 intrinsic subtype as a predictor of pathological complete response (pCR) in the breast following neoadjuvant dual HER2 blockade without chemotherapy in stage I-IIIA HER2 positive breast cancer. The underlying hypothesis for this trial was that the HER2-enriched breast tumor subtype (relative to luminal A, luminal B and basal) [1] provides independent predictive information additional to hormone receptor status. A total of 151 HER2 positive breast cancer patients received 18 weeks of dual neoadjuvant therapy with a combination of trastuzumab and lapatinib. Analysis of intrinsic subtype distribution using PAM50 was done at baseline and after the second week of treatment. Patients underwent surgery at the end of this period of induction therapy and received adjuvant systemic treatment at the discretion of the physician. The primary objective of this study was to assess whether the HER2-enriched subtype could predict pCR within the breast at the time of surgery. PAM50 analysis showed that at day 15 , the majority of tumors had become normal-like (48.9\%) or luminal A (27.5\%) in type with pCR rates in the breast of 46.9 and $11.9 \%$, respectively, for these two subtypes. The trial confirmed that the HER2-enriched subtype is a strong predictor of sensitivity to dual HER2 receptor blockade in the absence of chemotherapy. Moreover, PAM50 at baseline and after 2 weeks provides independent predictive information from hormone receptor status (note $50 \%$ of hormone receptor positive cases were HER2-enriched within this trial population).

'Cambridge Breast Unit, Addenbrookes Hospital \& University of Cambridge, Faculty of Medical Sciences, Anglia Ruskin University, Cambridge, UK

²Division of Surgical Oncology, Dale H. Dorn Chair in Surgery, University Of Texas Health Science Centre, San Antonio, TX, USA *Author for correspondence: Tel.: 00441223 586577; Fax: 00441223 586932; john.benson@addenbrookes.nhs.uk

\section{KEYWORDS}

- breast conserving surgery

- extended endocrine

therapy • mastectomy

- radiation treatment

- reconstruction

- San Antonio
Future $\because$
Medicine $\%$ part of 
The theme of anti-HER2 therapy was continued by Grazio Arpino (Naples, Italy) who presented results of the Phase II PERTAIN trial (NCT 01491737) which randomized postmenopausal women with hormone receptor and HER 2 positive metastatic or locally advanced breast cancer to either trastuzumab and an aromatase inhibitor (AI) or the same plus pertuzumab. The rationale for this combination of trastuzumab and pertuzumab was targeting of two different epitopes and minimizing the chance of HER2-ER bidirectional cross-talk which might be a mechanism of acquired resistance. A total of 258 patients were randomized to first-line treatment and about half had received prior treatment with chemotherapy. The primary end point of progression-free survival was increased by almost 12 months by addition of pertuzumab (27.1 vs 15.1 months; $\mathrm{p}=0.018$ ). The results of this study suggest that the combination of pertuzumab, trastuzumab and an AI is superior to trastuzumab and AI alone. Moreover, side effects were acceptable and no new safety issues arose.

Mothaffar Rimawi (Baylor College of Medicine, TX, USA) discussed results of the Phase III NSABP B53 trial (NRG ONCOLOGY B-53/S1207) which investigated cotargeting of HER 2 and endoplasmic reticulum (ER). Rates of $\mathrm{pCR}$ for tumors that are both ER and HER2 positive are lower than for ER negative and HER2-positive tumors suggesting that ER may antagonize action of anti-HER2 therapies [2]. Concurrent inhibition of ER alongside HER2 with (neoadjuvant) chemotherapy is hypothesized not to be antagonistic and will overcome any resistance with improved $\mathrm{pCR}$ rates. The B52 study randomized patients with ER/HER2 positive invasive ductal cancers $(>2 \mathrm{~cm}$; any size if node positive) to neoadjuvant docetaxel, carboplatin, trastuzumab and pertuzumab with or without estrogen deprivation (AI). The primary end point was pCR in the breast and axillary lymph nodes. There was a numerical increase in $\mathrm{pCR}$ for the group receiving estrogen deprivation ( 46 vs $41 \%$ ), but this was not statistically significant. It was concluded that addition of estrogen deprivation was not antagonistic and results supported a policy of de-escalation with potential avoidance of toxic chemotherapy and replacement with equally efficacious but less toxic endocrine therapies. However, it was less clear whether endocrine therapy given concurrently with pertuzumab plus trastuzumab can increase response rates. The trial failed to demonstrate the design aim of superiority, but could be interpreted as showing noninferiority. Usually endocrine therapy is given to hormone receptor positive patients as an adjuvant treatment. It was commented that this was essentially a negative trial and administration of an aromatase inhibitor may not be the best form of estrogen deprivation.

Reshma Jagsi (University of Michigan, MI, USA) discussed the impact of radiotherapy on complications and patient-reported outcome measures (PROMS) after breast reconstruction. Integration of post-mastectomy radiotherapy (PMRT) with breast reconstruction has proven challenging with limited evidence to guide treatment decisions and much variation in practice. PMRT induces tissues changes that can threaten viability of the reconstruction and final cosmetic outcomes with necessity for corrective surgery. The impact of PMRT on reconstruction is widely feared but poorly understood and current practice does not reflect shared decision-making informed by good quality data. In a prospective multicenter cohort study, irradiated patients ( $\mathrm{n}=$ 553) were compared with nonirradiated patients $(\mathrm{n}=1461)$ receiving either implant-based or autologous flap reconstruction. End points for this study were complications, reconstructive failures and PROMS. Mixed-effects regression models assessed impact of reconstruction type and radiotherapy and the interaction between these by adjustment of covariates. Patients having autologous reconstruction were more likely to be irradiated compared with implantbased techniques (38.3 vs $25.1 \%$; p < 0.001 ). Furthermore, irradiation was less common for immediate versus delayed procedures (82.6 vs 95.6\%; $\mathrm{p}<0.001)$. Multivariate analysis revealed that bilateral surgery and BMI were predictive of developing complications with significant interaction between receipt of radiotherapy and reconstruction type. Of note, at 2 years there was an increased chance of complications among irradiated implants compared with the nonirradiated group (odds ratio $=2.67 ; \mathrm{p}<0.001$ ), but there was no clear impact of PMRT on autologous reconstruction (odds ratio $=1.12 ; \mathrm{p}=0.67$ ) Predictably, reconstructive failure was higher for irradiated (11.4\%) than nonirradiated (3.4\%) patients. Autologous reconstruction was also associated with improved PROMS for psychosocial and physical domains. These data were considered helpful for making fully-informed 
and preference-concordant decisions on breast reconstruction.

A presentation by Pat Whitworth (Nashville Breast Center, TN, USA) examined the clinical utility of a ductal carcinoma-in situ (DCIS) biological risk profile for predicting recurrence after breast conserving surgery (BCS). Although randomized trials have confirmed that administration of radiotherapy after lumpectomy for localized forms of (screen-detected) DCIS reduces local recurrence, overall survival is the same for patients undergoing lumpectomy, lumpectomy and radiotherapy or mastectomy for pure forms of DCIS [3]. A biological risk signature was developed from integration of immunohistochemical markers and clinicalpathological factors to yield a continuous score from 1 to $10(\leq 3=\mathrm{LOW}$ SCORE; $>3=\mathrm{HIGH}$ SCORE). Biomarkers included PR, HER2, Ki-67, COX-2, p16, FOXA1 and SIAH2 and clinicopathological parameters were age, palpability, tumor size and margin status. A validation study recruited 986 patients with DCIS (no invasive component) between 1990 and 2007 among whom 694 underwent BCS (BCS alone $=78 ; \mathrm{BCS}$ with radiotherapy $=377)$. Almost half of cases were high nuclear grade DCIS $(42 \%)$ and about three quarters of these had necrosis (72\%). Whitworth emphasized that in the absence of clinicopathological factors, it is impossible to appropriately select patients for adjuvant radiotherapy after BCS. Overall rates of local recurrence for BCS alone and BCS with radiotherapy were 20 and $10 \%$. The biological risk score could divide patients treated with BCS $(+/$ - radiotherapy) into low $(\leq 3)$ and high risk (>3) groups with a HR: 1.87 (95\% CI: 1.033.38 ) with a $\mathrm{p}$-value of 0.038 . Those patients who did not receive radiotherapy and had a low score $(\leq 3)$ had a $10 \%$ risk of local recurrence at 10 years. Moreover, the assay identified patients (>3) with a relatively high (30\%) risk of relapse at 10 years without radiotherapy and this was reduced to $10 \%$ when these patients were treated with BCS and radiotherapy (RT). Therefore this assay identified a low-risk group which could potentially avoid radiotherapy and a higher risk group which is more likely to derive greater absolute benefits from radiotherapy following BCS. It was surmised that if high-risk patients alone were selected for radiotherapy, then a survival advantage might emerge.

Rowan Cheblowski (Los Angeles Biomedical Research Institute, CA, USA) provided an update on the Women's Health Initiative Dietary Modification study examining the influence of a low-fat diet on overall survival in breast cancer. Patients entered into this randomized controlled trial were reconsented after 8.5 years to allow a median period of intervention of 16.1 years. Dietary fat intake was reduced to $20 \%$ of total intake as a primary goal with a $40 \%$ decrease in energy supplies from fat in the first year. The recorded weight reduction for the dietary intervention group was $2.2 \mathrm{~kg}$ at 12 months ( $p$ $<0.001$ ) and the overall decrease in percentage calories from fat was $13.9 \%$ at year 1 and $8.2 \%$ at year $5(\mathrm{p}<0.001)$. In the cumulative follow-up period of 16.1 years, results revealed a nonsignificant decrease in risk of death from (i.e., directly attributable to) breast cancer (HR: 0.68 [0.43-0.94]; $\mathrm{p}=0.08$ ) and a statistically significant reduction in risk of death after breast cancer (HR: 0.65 [0.45-0.94]; p = 0.02). This dietary effect was more evident among women with a higher proportion of fat from total caloric intake $(>36.8 \%)$ at baseline and also among those with various other adverse lifestyle factors. Interestingly, it transpired that this dietary effect was more evident among women with a waist circumference of $\geq 88 \mathrm{~cm}$ and there was no benefit among women with lower percentage of calories from fat $(27.9 \%)$ nor women $>70$ years of age.

Anne Blaes (Masonic Cancer Center, MN, USA) discussed how AIs can modulate endothelial function and how this might be linked to early cardiovascular disease. Despite reductions in breast cancer specific mortality among women with operable hormone receptor positive disease, AIs have notable side effects including cardiovascular morbidity. Patients are now surviving longer due to efficacy of antitumor therapies but are more likely to die from cardiovascular disease than breast cancer. Endothelial dysfunction can be measured more directly based on hyperemia using EndoPAT which is a noninvasive technique based on measurement of elasticity in large and small arteries. Adverse EndoPAT scores are associated with increased risk of cardiovascular events and are independent of the Framingham risk score. It is proposed that women taking AIs will have worse EndoPAT scores due to a higher incidence of cardiovascular disease. A cross-sectional study was performed to test this hypothesis and recruited 25 healthy postmenopausal women (controls) and 36 postmenopausal women with 
locally advanced but curative breast cancer treated with aromatase inhibitors. A combination of biomarker and pulse-wave analysis with EndoPAT was undertaken. Measurement of large and small artery elasticity permitted calculation of the EndoPAT ratio (post/pre-occlusion) and pulse contour analysis. Results showed a trend toward reduced endothelial function based on large and small elasticity. Patients on AIs had a significantly reduced EndoPAT ratio of 0.8 compared with controls (2.7) ( $\mathrm{p}<$ 0.0001 ). The majority of breast cancer patients on AIs had abnormal EndoPAT ratios that are predictive of adverse cardiac events (acute coronary syndrome, myocardial infarction and cardiac death). Thus 39\% of patients with an abnormal EndoPAT (endothelial function) had a major cardiac event compared with $25 \%$ of those with normal endothelial function ( $\mathrm{p}=$ $0.024)$. These data support a significant reduction in endothelial function for patients receiving an AI. There is need for better biomarkers to predict risk of cardiovascular disease during AI therapy. Prospective evaluation of patients both before and after initiation of AI therapy is the next step with measurement of estradiol levels.

In the final General Session, Sonja Vliek (Netherlands Cancer Institute) presented first results of the TEAMIIB trial evaluating the efficacy and safety of adding ibandronate to adjuvant hormonal therapy in postmenopausal women with stage 1-III hormone receptor positive early breast cancer. The use of bisphosphonates in early breast cancer is controversial; a meta-analysis of more than 17,000 patients in 26 trials found a survival benefit for postmenopausal women only [4]. Estrogens are known to reduce the lifespan of osteoclasts and therefore the break on osteoclast activity is released in postmenopausal women. This in turn may render bisphosphonates more efficacious and any survival gains more apparent. Eligible patients were randomized after adequate surgery and radiotherapy to either adjuvant hormonal therapy alone or the same combined with ibandronate (50 mg daily) for 3 years with follow-up for 10 years. At a median follow-up of 4.6 years there was a trend for improved disease-free survival for ibandronate $(94.3 \% ; 29$ events) compared with control (90.8\%; 46 events) but this did not reach statistical significance (HR: 0.8 [0.58-1.10]; $\mathrm{p}=0.08)$. Fewer bone metastases as a first event occurred with bisphosphonates (1.2 vs 3.1\%), but once again not significant (HR: 0.59 [0.301.14]). Less than $1 \%$ of patients developed jaw osteonecrosis or severe impairment of renal function. Adherence at 3 years was 67 and 20\% of patients discontinued ibandronate therapy which may have underestimated the treatment effect. Moreover, this trial was slow to recruit with necessity for a power adjustment from 90 to $80 \%$ and extension of the accrual period to 6 years. Despite these shortcomings, results are concordant with other trials although it was conceded that this analysis may be premature.

The SABCS2016 featured work of many investigators not mentioned above but who have worked diligently to reduce to the burden of breast cancer. More importantly, the symposium continues to demonstrate the courage of thousands of women around the world who have participated in clinical trials and contributed to advances in the field of both clinical and translation research.

Financial \& competing interests disclosure

The authors have no relevant affiliations or financial involvement with any organization or entity with a financial interest in or financial conflict with the subject matter or materials discussed in the manuscript. This includes employment, consultancies, honoraria, stock ownership or options, expert testimony, grants or patents received or pending, or royalties.

No writing assistance was utilized in the production of this manuscript.

\section{References}

1 Prat A, Bianchini G, Thomas M et al. Research-based PAM-50 subtype predictor identifies higher responses and improved survival outcomes in HER2 positive breast cancer in the NOAH study. Clin. Cancer Res. 20(2, 511-521 (2014).

Bhargava R, Dabbs D, Beriwal Sushil et al. Semiquantitative hormone receptor level influences response to trastuzumab- containing neoadjuvant chemotherapy in HER2-positive breast cancer. Mod. Pathol. 24, 367-374 (2011).

3 Early Breast Cancer Trialists' Collaborative Group (EBCTCG). Effect of radiotherapy after mastectomy and axillary surgery on 10 year recurrence and 20 year breast cancer mortality: meta-analysis of individual patient data for 8135 women in 22 randomised trials. Lancet 383(9935), 2127-2135 (2014).
4 Rubinshstein R, Kuvin J, Soffler M et al. Assessment of endothelial cell function by non-invasive peripheral arterial tonometry predicts late cardiovascular adverse events. Eur. Heart J. 231, 1142-1148 (2010).

5 Early Breast Cancer Trialists' Collaborative Group (EBCTCG). Adjuvant bisphosphonate treatment in early breast cancer: metaanalyses of individual patient data from randomized trials. Lancet 386(10001), 1353-1361 (2015). 


\section{EXECUTIVE SUMMARY}

- The HER2-enriched subtype is a strong predictor of sensitivity to dual HER2 receptor blockade in the absence of chemotherapy.

- The combination of pertuzumab, trastuzumab and an aromatase inhibitor appears to be superior to trastuzumab and aromatase inhibitor alone.

- It remains unclear whether addition of endocrine therapy given concurrently with pertuzumab and trastuzumab can increase response rates.

- There is an increased chance of complications among breast reconstruction patients with irradiated implants at 2 years.

- There is no clear impact of irradiation on autologous tissue-based reconstruction.

- Rates of reconstructive failure are higher among implant-based compared with autologous flap-based reconstruction.

- A DCIS biological risk signature that integrates immunohistochemical markers and clinical-pathological factors has clinical utility.

- This DCIS signature can identify a low-risk group which could potentially avoid radiotherapy and a higher risk group with greater absolute benefits from radiotherapy.

- There is a nonsignificant decrease in risk of death from breast cancer and a significant reduction in risk of death after breast cancer from adoption of a low-fat diet ( $20 \%$ total caloric intake).

- There is a significant reduction in endothelial function for patients receiving aromatase inhibitors based on indirect measurements of elasticity in large and small arteries (EndoPAT).

- The use of bisphosphonates in early breast cancer patients remains controversial despite publication of a meta-analysis showing a survival benefit for postmenopausal women.

- Addition of ibandronate to adjuvant hormonal therapy in the TEAMIIB trial of post-menopausal stage I-III breast cancer showed a trend for improved disease-free survival with bisphosphonates, but no statistically significant benefit. 